\title{
Noncompliance: Das verdrängte Gesundheitsproblem
}

Stellen Sie sich vor, sie lesen in einer Zeitung folgende Nachricht: „Es gibt eine Krankheit, an der ca. 20 Millionen Bundesbürger leiden, an der pro Jahr 40000 Menschen sterben und die jährlich in Deutschland Kosten von 10 Milliarden Euro verursacht. Diese Krankheit ist seit Jahrzehnten bekannt, man weiß auch, wie man sie effektiv behandeln kann, doch eine Behandlung wird hierzulande nicht angeboten, weil die Kostenträger sie nicht bezahlen“.

Wie wäre Ihre Reaktion? Skepsis, Empörung oder zumindest Neugier, um welche Krankheit es sich dabei handeln könnte? Die Rede ist natürlich von der „Volkskrankheit“ Noncompliance, einem nahezu ubiquitären Verhaltensmuster, das natürlich keine Krankheit im engeren Sinne darstellt, dessen Folgen aber Krankheiten verschlimmern bzw. neu hervorrufen können. Und wenn man den Kostenträgern die Relevanz dieses Problems verdeutlichen will, ist die Analogie vielleicht hilfreich.

Noncompliance ist praktisch bei jeder Langzeitmedikation zu beobachten. Dass sie deshalb eines der wichtigsten Gesundheitsprobleme überhaut darstellt, ist seit langem bekannt und bekannt ist auch, wie Noncompliance wirksam verhindert werden kann. Complianceverbessernde Maßnahmen werden jedoch trotzdem in der Regelversorgung nicht angeboten, da sie entweder überhaupt nicht oder nicht kostendeckend honoriert werden.

Dass das oben Gesagte natürlich besonders auch für Erkrankungen aus unserem Fachgebiet gilt und die Noncompliance-Raten hier z.T. sogar noch über 50\% (!) liegen, daran werden wir im ersten Artikel dieses Heftes von Ute Seemann schmerzhaft erinnert. Im klinischen Alltag neigt man dazu, die kränkende Tatsache zu verdrängen, dass die Hälfte unserer Patienten die von uns empfohlene und dringend indizierte Langzeitbehandlung nicht durchführen. Die Verdrängung wird dadurch gefördert, dass wir noncompliante
Patienten häufig aus den Augen verlieren, da diese oft nicht mehr in die Praxis kommen sondern still und leise aus dem Versorgungssystem verschwinden - bis sie wegen ihres nächsten Rückfalls wieder im Krankenhaus auftauchen.

Eine Lösung des Compliance-Problems scheint jetzt erstmals in greifbare Nähe gerückt. Zum einen gibt es zahlreiche neue und erfolgversprechende Verfahren zur Compliance-Verbesserung, über die Michaela Stiegler sowie Christine Rummel-Kluge und Wulf-Peter Hansen in ihren Artikeln berichten. Zum andern gibt es endlich eine Möglichkeit, all diese complianceverbessernden Maßnahmen von den Krankenkassen angemessen honoriert zu bekommen. Dass wir bisher in der Regelversorgung kaum complianceverbessernde Maßnahmen angeboten haben, konnten wir immer auch damit entschuldigen, dass gerade im ambulanten Bereich dafür keine, d.h. keine bzw. keine angemessen honorierten Gebührenziffern vorgesehen waren.

Diese „Ausrede“ gilt ab sofort nicht mehr. Im Rahmen des Gesundheitsreformgesetzes wurde die Möglichkeit geschaffen, Compliance-Programme durch Direktverträge mit den Krankenkassen (z.B. nach § 140 SGB V „Integrierte Versorgung“) zusätzlich zu den geltenden Budgets angemessen zu finanzieren. Da diese Möglichkeit von Psychiatern und Neurologen bisher noch kaum genutzt wurde, wird in diesem Heft am Beispiel eines seit 3 Jahren erfolgreich laufenden Integrierten Versorgungsmodells („Münchner Modell“") dargestellt, wie man ein solches Compliance-Programm bei neuropsychiatrischen Erkrankungen konzipieren und mit den Krankenkassen vertraglich vereinbaren kann.

Sollte es damit nach jahrzehntelangen Versäumnissen auf diesem Gebiet doch noch gelingen, das größte Gesundheitsproblem - die Noncompliance - einer Lösung oder zumindest einer Besserung näher zu bringen? Es wäre - auch im Interesse unserer Patienten - sehr zu hoffen. 